\title{
Short-term clinical outcome and dosimetric comparison of tandem and ring versus tandem and ovoids intracavitary applicators
}

\author{
John K. Ma, MD, PhD', Waleed F. Mourad, MD², Robert Allbright, MD', Satyaseelan Packianathan, MD, PhD', \\ Leslie M. Harrell, DO', Edmund Chinchar, MD², Alex Nguyen, MS', Srinivasan Vijayakumar, MD' \\ 'Department of Radiation Oncology, ${ }^{2}$ Department of Medicine, University of Mississippi, Jackson, MS, USA
}

\begin{abstract}
Purpose: To compare the short-term toxicity and dosimetry of tandem and ring (TR), and tandem and ovoid (TO) applicators in treatment of gynecologic malignancy.

Material and methods: Following pelvic external beam radiation therapy (EBRT), a total of 52 computed tomography-based plans from 13 patients with cervical cancer (FIGO IB2-IIIB) were evaluated for HDR brachytherapy. Prescription was 7 Gy to the ICRU point A for four weekly fractions. Gastrointestinal and genitourinary toxicities were evaluated. Clinical target volume (CTV) and organs at risk were delineated on CT scans. Bladder, rectum, and sigmoid mean doses and $\mathrm{D}_{2 c c}$ were calculated. Treatment time and irradiated tissue volume were compared. Percent of CTV receiving $100 \%\left(\mathrm{CTV}_{100 \%}\right)$ of the prescribed dose as well as the percent of the prescription dose covering $90 \%$ of the CTV $\left(\mathrm{D}_{90}\right)$ were evaluated.

Results: Gastrointestinal and genitourinary toxicities were not different between TO and TR applicators. No significant differences in the dose to the right and left point A, or the left point $B$ were observed. TO delivered a higher dose to right point B. Organs at risk doses were similar between the two applicators, except mean rectal dose was lower for TO applicator. Overall, TO treats a larger tissue volume than TR. Mean treatment time was shorter for TR. Tumor coverage $\left(\mathrm{D}_{90}\right.$ and $\left.\mathrm{CTV}_{100 \%}\right)$ was equivalent between $\mathrm{TO}$ and TR applicators.

Conclusion: Although TO treats a larger tissue volume than TR, short-term toxicities and tumor coverage are sim-
\end{abstract} ilar. Long-term clinical outcomes will be elucidated with longer follow up period.

J Contemp Brachytherapy 2015; 7, 3: 218-223 DOI: $10.5114 /$ jcb.2015.51853

Key words: brachytherapy, cervical cancer, dosimetry, radiation toxicity.

\section{Purpose}

Intracavitary brachytherapy (ICBT) is an essential part of the treatment of cervical cancer and has been shown to improve outcomes [1-3]. Secondary to sharp dose gradient, advantages in dose optimization, a lower radiation dose to personnel, and shortened hospital stay as an outpatient procedure, high-dose-rate (HDR) brachytherapy has been widely used [4]. Remote afterloading HDR brachytherapy became popular in the 1960s [5,6]. According to the American Brachytherapy Society survey in 1995, 24\% of institutions use HDR brachytherapy in the treatment of cervical cancer [7]. Prevalence of HDR utilization has increased in recent years [8].

The two most common HDR ICBT applicators used in treatment of cervical cancers are the tandem and 2 ovoids (TO) and the tandem and ring (TR) [4]. TR was introduced after TO to render additional benefits to patients such as increased patient comfort, feasibility in patients with narrow vagina, applicability in women with obliterated vaginal fornices, and improved reproducibility $[9,10]$. Previous studies have examined the dosimetric profiles of TO and TR via orthogonal radiographs [11] and computed tomography (CT) images [12]; however, no correlations have been made in relationship to clinical outcomes such as short-term toxicity. Erickson et al. compared the dose distributions of TO with those of TR applicator [11] via orthogonal radiographs. Specific point dose comparisons were made. However, no isodose volume comparisons were made secondary to a lack of post insertion computed tomography $(\mathrm{CT})$ verification. In their study, the results showed a larger isodose volume in TO than TR by comparing the height and width of isodose curve in a single dimension at a time. Studies have also addressed the
Address for correspondence: John K. Ma, MD, PhD, Department of Radiation Oncology, University of Mississippi, Jackson, MS, USA, 2500 North State Street, Jackson, MS 39216, USA, phone: +1 601 5198162, fax: +1 601 8156876, « e-mail: johnma33@hotmail.com
Received: 27.01.2015

Accepted: 16.04 .2015

Published: 25.06.2015 
spatial reproducibility of TO and TR applicators $[7,13,14]$. In the study by Levin et al., dosimetric comparisons of $\mathrm{TO}$ and TR were investigated using $\mathrm{CT}$ guided volume determination after applicator insertion in patients with stage II-IV cervical cancer [12]. The authors showed that even though the optimization point doses were similar between TO and TR applicators, TO applicator clearly exhibited a larger isodose volume in comparison to TR applicator. No correlation between the dosimetric profiles and clinical outcome were made.

The purpose of the present study is to correlate short term clinical outcomes with dosimetric profile comparison between TO and TR applicators using CT guided planning system in the setting of multifractionated HDR ICBT for gynecologic malignancy.

\section{Material and methods}

\section{Patients}

Between 2011 and 2013, a total of thirteen women with cervical cancer, with International Federation of Gynecology and Obstetrics (FIGO) stage IB2-IIB were treated with HDR ICBT with Ir $^{192}$ sources. Patients were identified retrospectively. All patients were treated with external beam radiotherapy (EBRT) consisting of 45 Gy in 25 fractions to the pelvis prior to ICBT. Parametrial boost of 5.4 Gy in 3 fractions were given to those patient who warranted it. In the present study, eight patients were treated with TO applicator and five patients with TR applicator. HDR brachytherapy consisted of 7 Gy per fraction given one week apart for a total of four fractions.

\section{Intraoperative procedure}

The selection of applicator was based on preference and the experience of the attending physician. One physician used TO applicators exclusively, whereas the other radiation oncologist preferred TR applicators. The first insertion of ICBT applicators was performed while patients were under anesthesia in the operating room. A Foley catheter filled with saline solution was inserted into the bladder. Sounding the uterus, cervical dilation, and Smitt sleeve placement typically took place in the OR in the presence of a gynecologic oncologist. After applica- tor placement was verified, vaginal packing was applied. The subsequent applicator insertions were performed in the outpatient clinic with the patient placed under conscious sedation. The TO and TR applicators are shown in Figure 1. Small ovoids were utilized in the 32 TO insertions. Vaginal packing was applied subsequently for TO insertions, both anterior and posterior to the tandem to minimize bladder and rectal doses. Rectal tube was inserted, after which rectal contrast was injected. For TR insertions, $60^{\circ}$ ring and rectal retractor were utilized in all TR patients.

\section{Computed tomography simulation}

After each insertion and subsequent recovery from sedation, the patient underwent CT simulation ( $3 \mathrm{~mm}$ slice) via Philips Brilliance CT simulator (Philips Healthcare, Inc., Andover, MA) for CT based treatment planning using the software Brachyvision (Varian Medical Systems, Inc., Palo Alto, CA). All patients were scanned in the supine position.

\section{Treatment planning and target delineation}

Each patient was prescribed 7 Gy to ICRU point A for a total of four fractions. After delineation of organs at risk (OAR), a 3D treatment plan was generated. For each brachytherapy fraction, a new plan was generated for each insertion to account for inter-fraction positional or anatomical changes. The rectum, bladder, and sigmoid colon were contoured on each CT slice. Bladder, rectum, and sigmoid doses and $\mathrm{D}_{2 \mathrm{cc}}$ were calculated. In order to modify the isodose curve to bring OAR $\mathrm{D}_{2 \mathrm{cc}}$ under GEC-ESTRO recommendations, optimization was achieved by adjusting the radioactive source loading patterns, dwell positions, and dwell times. Treatment fractions were delivered with a HDR Ir $^{192}$ source afterloading system.

Retrospectively, CTV was contoured using the CT based high risk CTV delineation guidelines by Viswanathan et al. [15]. Mean treatment time and total volume of all tissues receiving $95 \%\left(\mathrm{~V}_{95 \%}\right), 85 \%\left(\mathrm{~V}_{85 \%}\right), 50 \%\left(\mathrm{~V}_{50 \%}\right)$, and $20 \%\left(\mathrm{~V}_{20 \%}\right)$ of the brachytherapy prescription dose were compared. Percent of CTV receiving $100 \%\left(\mathrm{CTV}_{100 \%}\right)$ of the prescribed dose as well as the percent of prescrip-
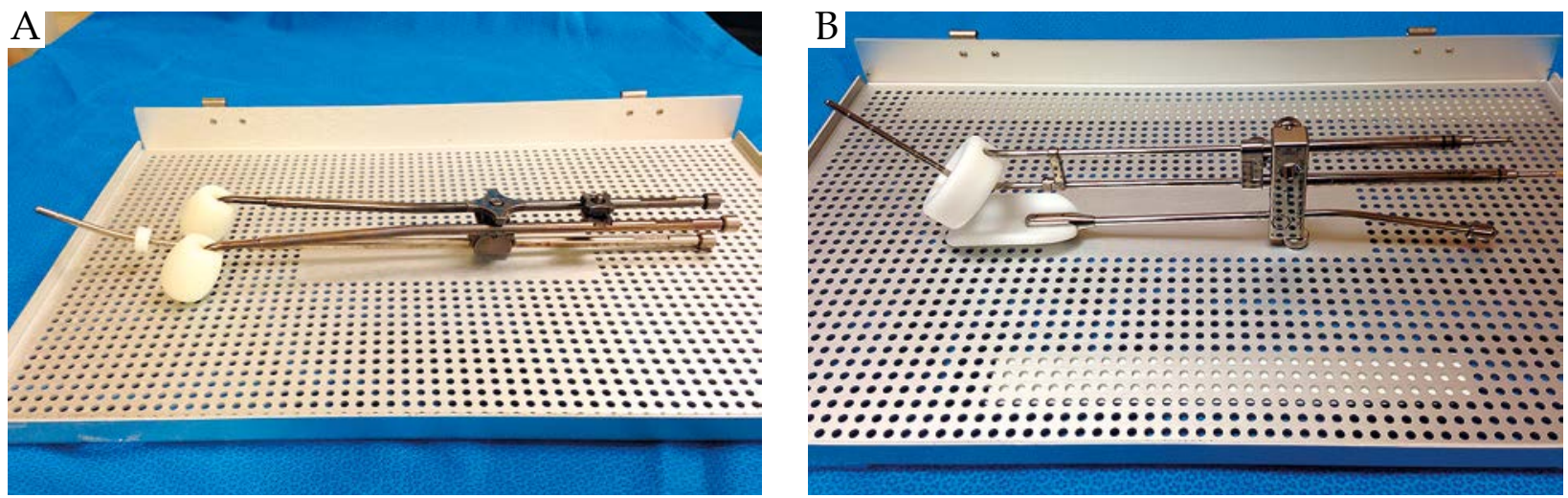

Fig. 1. A) Tandem and ovoids applicator assembled. B) Tandem and ring applicator assembled 

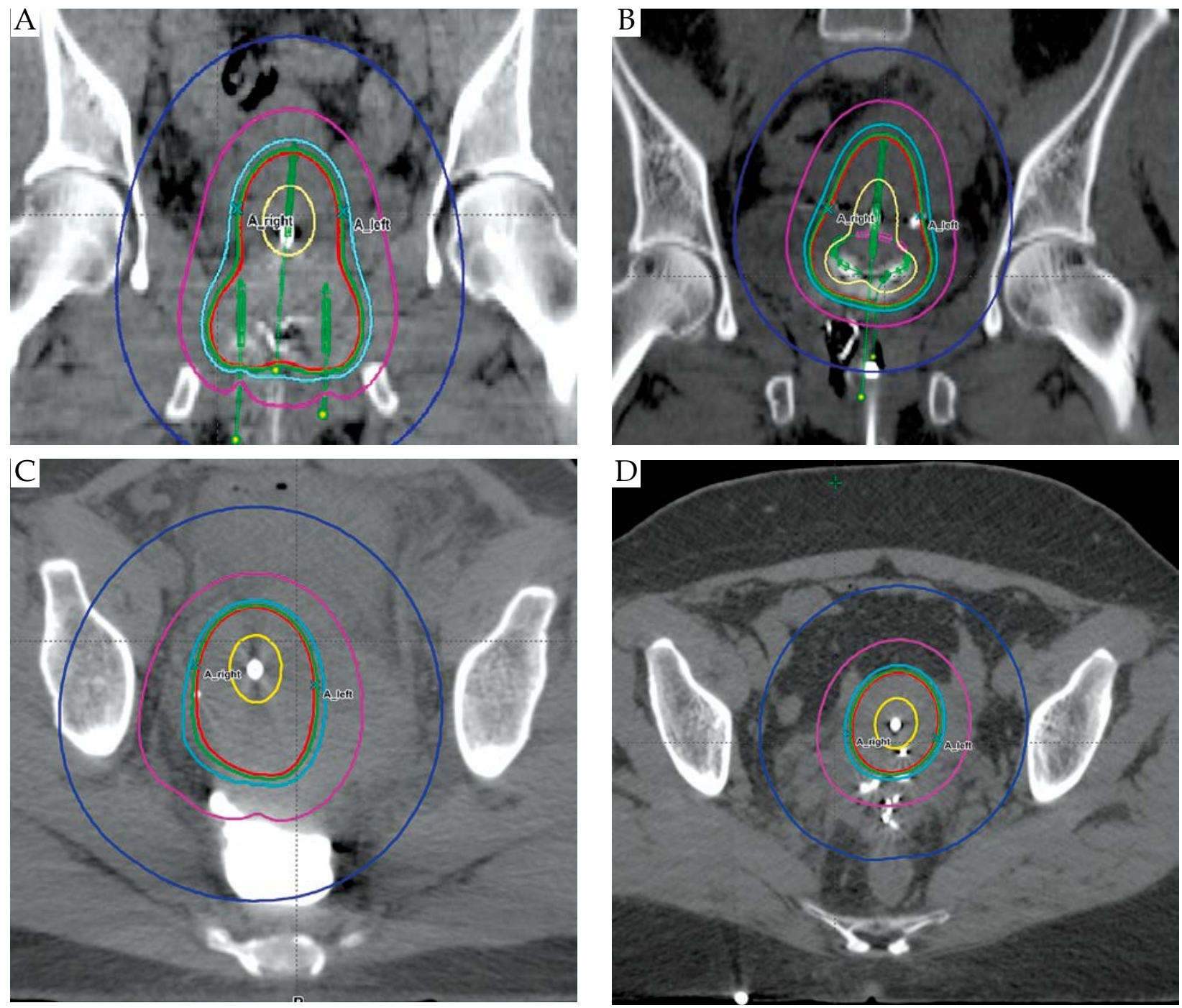

Fig. 2. Coronal and axial views of isodose distribution and prescription points A (left and right). Coronal view of tandem and ovoids isodose lines (A). Coronal view of tandem and ring isodose lines (B). Axial view of tandem and ovoids isodose lines (C). Axial view of tandem and ring isodose lines (D). Isodose lines as a percentage of the prescribed dose to point A: yellow (200\%), red $(100 \%)$, green $(95 \%)$, cyan (85\%), magenta $(50 \%)$, and blue (20\%). Isodose lines are shown for small ovoids in TO applicators

tion dose covering $90 \%$ of the CTV $\left(\mathrm{D}_{90}\right)$ were evaluated. Examples of the isodose lines are shown in the coronal plane for TO (Fig. 2A) and TR (Fig. 2B) as well as in the axial plane for TO (Fig. 2C) and TR (Fig. 2D) applicators.

\section{Clinical outcome measurement}

Gastrointestinal toxicity and genitourinary toxicity were evaluated weekly and graded according to RTOG toxicity criteria. The final evaluation for short term toxicity took place one month after the last insertion.

\section{Statistics}

Mean values, standard deviations, and standard error of mean were calculated from $32 \mathrm{TO}$ and $20 \mathrm{TR}$ applications. Because we could not assume whether TO or TR values would be larger or smaller, two tail $t$ test was utilized to calculate $p$-value.

\section{Results}

All patients received four fractions of HDR brachytherapy for a total of 52 treatments (32 TO and 20 TR). Dosimetric results of the 52 applications and CT-based plans are presented in Table 1. Of the measured parameters, the dose to the right point $\mathrm{B}$ was significantly different between TO and TR. The mean dose to the right point $B$ was higher in TO than TR. Although the rectal $\mathrm{D}_{2 c c}$ were statistically similar between TO and TR, the mean rectal dose in TR was lower than TO. Given that the primary optimization goal was to deliver the prescription dose to points A, dosimetric results were similar for most parameters between the two applicators. Optimization goals were made to achieve GEC ESTRO dose constraints to bladder, rectum, and sigmoid colon. Hence, doses were intentionally minimized to the OAR contoured, resulting in no statistical differences seen in the dose to points $A$, 
Table 1. Dosimetric parameters for tandem and ovoids (TO) and tandem and ring (TR) applicators

\begin{tabular}{lccc} 
& TO (cGy) & TR (cGy) & $p$ value \\
\hline Right point A mean dose & $693 \pm 17$ & $686 \pm 39$ & 0.55 \\
\hline Left point A mean dose & $691 \pm 21$ & $673 \pm 42$ & 0.16 \\
\hline Right point B mean dose & $233 \pm 56$ & $179 \pm 39$ & 0.00086 \\
\hline Left point B mean dose & $214 \pm 61$ & $198 \pm 48$ & 0.32 \\
\hline Rectum mean dose & $223 \pm 65$ & $162 \pm 35$ & 0.00018 \\
\hline Rectum $\mathrm{D}_{2 c c}$ & $408 \pm 111$ & $360 \pm 105$ & 0.148 \\
\hline Bladder mean dose & $329 \pm 112$ & $328 \pm 75$ & 0.97 \\
\hline Bladder $\mathrm{D}_{2 c c}$ & $438 \pm 196$ & $397 \pm 98$ & 0.35 \\
\hline Sigmoid mean dose & $184 \pm 61$ & $185 \pm 47$ & 0.95
\end{tabular}

Mean value and standard deviations were calculated from 32 TO and 20 TR treatments. $p$ values were generated using two tail t test. $D_{2 c c}-$ minimum dose to the most exposed $2 \mathrm{~cm}^{3}$

the $\mathrm{D}_{2 \mathrm{cc}}$ rectum, the mean dose, and $\mathrm{D}_{2 \mathrm{cc}}$ bladder, or the mean dose and $D_{2 c c}$ sigmoid.

Treatment volumes and durations are presented in Table 2. $\mathrm{V}_{95 \%}, \mathrm{~V}_{85 \%}, \mathrm{~V}_{50 \%}$, and $\mathrm{V}_{20 \%}$ were all significantly higher for TO than for TR $(p<0.018)$. Qualitatively, this can be seen in Figure 2. The typical isodose line volumes $(95 \%, 85 \%, 50 \%$, and $20 \%)$ appear larger in TO than TR in both the coronal and axial dimensions. The volume enclosed by the prescription surface as well as the shape of the dose distributions were different between the two applicators. The total treatment time was significantly longer for TO vs TR applicators $(p<0.0001)$. Despite the larger isodose volume seen in TO, the percent of the CTV that received $100 \%$ of the prescription dose $\left(\mathrm{CTV}_{100 \%}\right)$ and the percent of prescription dose covering $90 \%$ of the CTV $\left(\mathrm{D}_{90}\right)$ were not statistically different between TO and TR applicators. For dose distributions in regards to TO applicators, the above dosimetric parameters are described for applicators using small ovoids.

Clinically, there were no statistical differences in the RTOG acute $\geq$ grade 2 GI or $\geq$ grade 2 GU toxicities seen between TO and TR (Table 2). Two patients in the TO group experienced acute $\geq$ grade 2 GI toxicity, whereas one patient in the TR group experienced acute $\geq$ grade 2 GI toxicity. No vaginal fibrosis or stenosis was detected in either group during short term follow up. No patient experienced $\geq$ grade $2 \mathrm{GU}$ toxicity in either group.

\section{Discussion}

Tandem and ring HDR brachytherapy has been gaining wider utilization secondary to increased patient comfort, ease of insertion, inter fractional consistency, and applicability in patient with narrow vagina [9]. In the current study, both applicators were able to satisfy optimization goals and OAR tolerance, but they produced very different treatment volumes and treatment times. This study kept optimization goal to 700 cGy at point A for every fraction to minimize inter-fraction differences and maintain consistency between TO and TR doses. Within the same setting, when attempting to deliver the same prescription dose to point $\mathrm{A}$, TO applicator delivers the same dose as TR to a significantly larger volume with longer treatment duration. This difference may be due to the relative changes in the anatomy, in relation to the location of the treatment volume and critical organs as the applicator is inserted. As seen qualitatively in Figure 1, the assembled TO apparatus exhibits a wider distribution at the level of the ovoids as well as thicker anterior posterior distribution. Hence, to achieve the same prescription dose to point $A$, the overall treated volume is higher for TO. The results are in agreement with the dosimetric findings of earlier studies using 2D radiographs [11] as well as 3D CT volumes [12]. The two applicators are not dosimetrically equivalent. Alternatively, differences in the techniques between two different radiation oncologists may account for the dosimetric differences, since each radiation oncologist clearly exhibited preference for one type of applicator over the other. Intuitively, the shape of the adjacent anatomy and hence the CTV delineation, depend on the applicator type used. It is interesting that the right point B dose is higher in TO than TR. Since the dose to point B is not an optimization goal, the results show fundamental physical differences between the two applicators. Since point A is defined in relationship to the tandem whereas point $B$ is defined according to the anatomical midline, the differences seen in the point $B$ dose may have resulted from fundamental differences or asymmetry in the position of the tandem to that of the ovoids. The positioning of the ovoids largely depend on the shape and symmetry of the vaginal fornix. During insertion, the tandem does not always bisect the distance between the ovoids in the midline of the coronal plane. The innate design of TO applicator allows a greater degree of motion between the tandem and the ovoids than between the tandem and the ring in the assembled TR applicator. Therefore, the differences seen in the point $\mathrm{B}$ dose between TO and TR applicators may be due to the differences in the design that allows more freedom of movement in TO applicator.

The more important question is addressing the clinical relevance of such dosimetric differences. Point A for both TO and TR have been optimized to 7 Gy; however, point A dose not necessarily reflect the dose to the tar- 
Table 2. Volumes of all tissues that received various doses, target volume receiving prescription dose, total duration of treatment, and acute toxicities for tandem and ovoids (TO) and tandem and ring (TR) treatments

\begin{tabular}{lccc} 
& TO & TR & $p$ value \\
\hline $\mathrm{V}_{95 \%}(\mathrm{cc})$ & $123 \pm 25$ & $104 \pm 26$ & 0.018 \\
\hline $\mathrm{V}_{85 \%}(\mathrm{cc})$ & $147 \pm 30$ & $123 \pm 31$ & 0.012 \\
\hline $\mathrm{V}_{50 \%}(\mathrm{cc})$ & $329 \pm 70$ & $267 \pm 68$ & 0.0050 \\
\hline $\mathrm{V}_{20 \%}(\mathrm{cc})$ & $1187 \pm 253$ & $972 \pm 242$ & 0.0064 \\
\hline $\mathrm{CTV} \mathrm{V}_{100 \%}(\%)$ & $55 \pm 14$ & $58 \pm 11$ & 0.45 \\
\hline $\mathrm{D}_{90}(\%)$ & $56 \pm 16$ & $55 \pm 12$ & 0.77 \\
\hline Mean treatment time (seconds) & $848 \pm 188$ & $673 \pm 73$ & 0.00005 \\
\hline$\geq$ grade 2 Gl toxicity (\%) & 20 & 25 & 1.00 \\
\hline$\geq$ grade 2 GU toxicity (\%) & 0 & 0 & $\mathrm{NS}$
\end{tabular}

Mean value and standard deviations were calculated from 32 TO and 20 TR treatments; $p$ values were generated using two tail t test.

CTV - clinical target volume, GI - gastrointestinal, GU - genitourinary, CTV $100 \%$ - percent of CTV receiving $100 \%$ of dose, $D_{90}$ - percent of the prescription dose covering $90 \%$ of the CTV; $V_{95 \%}, 85 \%, 50 \%$, 20\% - target volume receiving at least $95,85,50$ or $20 \%$ of prescription dose

geted volume. Hence, determining the doses to the CTV, delineated via CT based contour according to guidelines by Viswanathan et al. [15], is essential to the comparison of future clinical outcomes such as locoregional control. In this setting, the CT based planning modality appears inferior to MRI based images in terms of distinguishing differences between the uterus, cervix, bladder, rectum, and the tumor. Though MRI based consensus guidelines are available for delineation of clinical target volumes for intensity modulated pelvic radiotherapy [13], the applicators utilized were not compatible with MRI and no MRI simulators were readily available. Therefore, the contours of CT and OAR were accomplished via CT based guidelines.

Despite the aforementioned, the total treatment volume differences seen between $\mathrm{TO}$ and $\mathrm{TR}, \mathrm{CTV}_{100 \%}$, and $\mathrm{D}_{90}$ were not different between the two applicators. Under the same conditions, one would expect the same dose to the targeting volume $\left(\mathrm{CTV}_{100 \%}\right)$ and $\mathrm{D}_{90}$ would result in the same LRC, an outcome that will be elucidated with longer follow up. At present, a larger treatment volume by TO applicator did not translate to differences in $\mathrm{CTV}_{100 \%}$ or $\mathrm{D}_{90}$. Nor did the larger treatment volume translate to higher acute GI or GU toxicity from TO applicator. This is not surprising since the optimization goals already accounted for bladder, rectum, and sigmoid colon doses as exhibited by a lack of statistically significant differences in GEC-ESTRO recommended $\mathrm{D}_{2 c c}$ to the bladder, rectum, or sigmoid colon between the two applicators. As such, no differences in the GI or GU toxicities were observed between patients who were treated with TO vs. TR.

Given that it is the practice at our institution to prescribe the dose to point $A$, we acknowledge the limitation of retrospectively outlining the target volumes after the completion of therapy. Furthermore, there are limitations to target delineation via $\mathrm{CT}$ simulation as compared to MRI, which is recommended by GEC-ESTRO [15]. However, due to logistic reasons, MRI is not readily available after insertion. Hence, $\mathrm{CTV}_{100 \%}$ and $\mathrm{D}_{90}$ appear low when one attempts to impose $2 \mathrm{D}$ optimization unto a $3 \mathrm{D}$ volu- metric data set. We have shown that although there were differences in the overall treatment volumes between the applicators, the same prescription dose to point $\mathrm{A}$ in $\mathrm{TO}$ and TR result in the same coverage to the clinical targeting volume with equivalent acute clinical toxicity.

\section{Conclusions}

The results show that although the two applicators deliver similar prescribed dose to point $\mathrm{A}$ and maintain critical organ doses below tolerance, they treat different volumes. Higher amounts of normal tissue are treated by the TO applicator, though the short-term toxicities are not increased. Even though the TR applicator covers less total tissue volume, dose distribution to the tumor volume is not compromised. Longer follow up period is required to evaluate long-term clinical outcomes such as loco-regional control, disease free survival, overall survival, longterm toxicity, quality of life, and correlation to differences seen in such parameters as $\mathrm{V}_{95 \%}, \mathrm{~V}_{85 \%}, \mathrm{~V}_{50 \%}$, and $\mathrm{V}_{20 \%}$ between TO and TR applicators.

\section{Disclosure}

Authors report no conflict of interest.

\section{References}

1. Coia L, Won M, Lanciano R et al. The Patterns of Care Outcome Study for cancer of the uterine cervix. Results of the Second National Practice Survey. Cancer 1990; 66: 2451-2456.

2. Lanciano RM, Won M, Coia LR et al. Pretreatment and treatment factors associated with improved outcome in squamous cell carcinoma of the uterine cervix: a final report of the 1973 and 1978 patterns of care studies. Int J Radiat Oncol Biol Phys 1991; 20: 667-676.

3. Logsdon MD, Eifel PJ. FIGO IIIB squamous cell carcinoma of the cervix: an analysis of prognostic factors emphasizing the balance between external beam and intracavitary radiation therapy. Int J Radiat Oncol Biol Phys 1999; 43: 763-775.

4. Nag S, Erickson B, Thomadsen B et al. The American Brachytherapy Society recommendations for high-dose-rate brachytherapy for carcinoma of the cervix. Int J Radiat Oncol Biol Phys 2000; 48: 201-211. 
5. Henschke UK, Hilaris BS, Mahan GD. Remote Afterloading with Intracavitary Applicators. Radiology 1964; 83: 344-345.

6. O'Connell D, Howard N, Joslin CA et al. A new remotely controlled unit for the treatment of uterine carcinoma. Lancet 1965; 2: 570-571.

7. Bahena JH, Martinez A, Yan D et al. Spatial reproducibility of the ring and tandem high-dose rate cervix applicator. Int J Radiat Oncol Biol Phys 1998; 41: 13-19.

8. Gaffney DK, Du Bois A, Narayan K et al. Practice patterns of radiotherapy in cervical cancer among member groups of the Gynecologic Cancer Intergroup (GCIG). Int J Radiat Oncol Biol Phys 2007; 68: 485-490.

9. Wolli, M, Kagan A, Olch A et al. Comparison of the ring applicator and the Fletcher applicator for HDR gynaecological brachytherapy. Selectron Brach J 1991; 2: 25-27.

10. Nair MT, Cheng MC, Barker A et al. High dose rate (HDR) brachytherapy technique: for carcinoma of uterine cervix using Nucletron applicators. Med Dosim 1995; 20: 201-207.

11. Erickson B, Jones R, Rownd J et al. Is the tandem and ring applicator a suitable alternative to the high dose rate selectron tandem and ovoid applicator. J Brachyther Int 2000; 16: 131-144.

12. Levin D, Menhel J, Rabin T et al. Dosimetric comparison of tandem and Ovoids vs. tandem and ring for intracavitary gynecologic applications. Med Dosim 2008; 33: 315-320.

13. Mai J, Erickson B, Rownd J et al. Comparison of four different dose specification methods for high-dose-rate intracavitary radiation for treatment of cervical cancer. Int J Radiat Oncol Biol Phys 2001; 51: 1131-1141.

14. Tan LT, Coles CE, Hart C et al. Clinical impact of computed tomography-based image-guided brachytherapy for cervix cancer using the tandem-ring applicator - the Addenbrooke's experience. Clin Oncol (R Coll Radiol) 2009; 21: 175-182.

15. Viswanathan AN, Dimopoulos J, Kirisits C et al. Computed tomography versus magnetic resonance imaging-based contouring in cervical cancer brachytherapy: results of a prospective trial and preliminary guidelines for standardized contours. Int J Radiat Oncol Biol Phys 2007; 68: 491-498. 